Supporting Information for

\title{
Escherichia Coli Swim back Towards Stiffer Polyetheylene glycol Coatings, increasing Contact in Flow
}

Molly K. Shave, ${ }^{1}$ Zhou Xu, ${ }^{2}$ Vishnu Raman, ${ }^{3}$ Surachate Kalasin, ${ }^{1}$ Mark T. Tuominen, ${ }^{2}$ Neil S. Forbes, ${ }^{3}$ Maria M. Santore*1

1. Department of Polymer Science and Engineering, University of Massachusetts, Amherst, MA 01003

2. Department of Physics, University of Massachusetts, Amherst, MA 01003

3. Department of Chemical Engineering, University of Massachusetts, Amherst, MA 01003

\section{Plasmid for Upregulating Flagella.}

To upregulate flagella growth the flhDC gene was amplified using PCR from the Salmonella (SL1344) genomic DNA using the following primers: Forward aaaaaCCATGGgtTAATAAaaggaggaatatatATGCATACATCCGAGTTGCTAAAACA ReverseaaaaaCTCGAGaaaaTTAAACAGCCTGTTCGATCTGTTCAT (Invitrogen). The PCR product was digested with NcoI-HF and XhoI-HF (New England Biolabs). The PBAD-his-myc A plasmid (Invitrogen) was also digested with NcoI-HF and XhoI-HF (NEB). The digested vector and PCR product were mixed together at a ratio of 50 nanograms of plasmid backbone to 500 nanograms of PCR product and ligated together with T4 DNA ligase in ligase buffer. The resulting plasmid (pflhDC) was transformed into DH5alpha E. Coli (CGSC). To allow fluorescent detection of bacteria, the plasmid also contained a green fluorescent protein expression cassette. The GFPmut3 protein was amplified through PCR using the following primers:

Forward:

aaaaaGCGGCCGCTTTACACTTTATGCTTCCGGCTCGTATAATGAATTCAAAAAAAAGGAGGA AAAAAAATGAGTAAAGGAGAAGAACTTTTCA

and Reverse:

aaaaaGCGGCCGCTTATTTGTATAGTTCATCCATGCCAT (Invitrogen).

The pflhDC plasmid and EGFP PCR product were digested with NotI-HF (NEB). After using a PCR cleanup column (Zymo Research), the NotI-HF digested PCR product and pflhDC plasmid were ligated together with T4 DNA ligase in ligase buffer to create the pflhDC+EGFP plasmid. The resulting plasmid was transformed into DH5alpha. 


\section{Motility Assay.}

A plate motility assay was used to confirm the motility of the strains. This assay establishes bacterial motility based on the observed colony expansion as a result of cells swimming or swarming outward from a small inoculated region at the center of an agar plate. ${ }^{1,2}$ Figure SI-1A presents images of the plates 72 hours after inoculation. The swimmers showed substantial mobility, reaching the edge of the plate in approximately 24 hours, as summarized in Figure SI-1B. Conversely, the persistently small size of the non-motile colony, with only slight colony expansion due to crowding, is consistent with the intended lack of mobility in this strain.

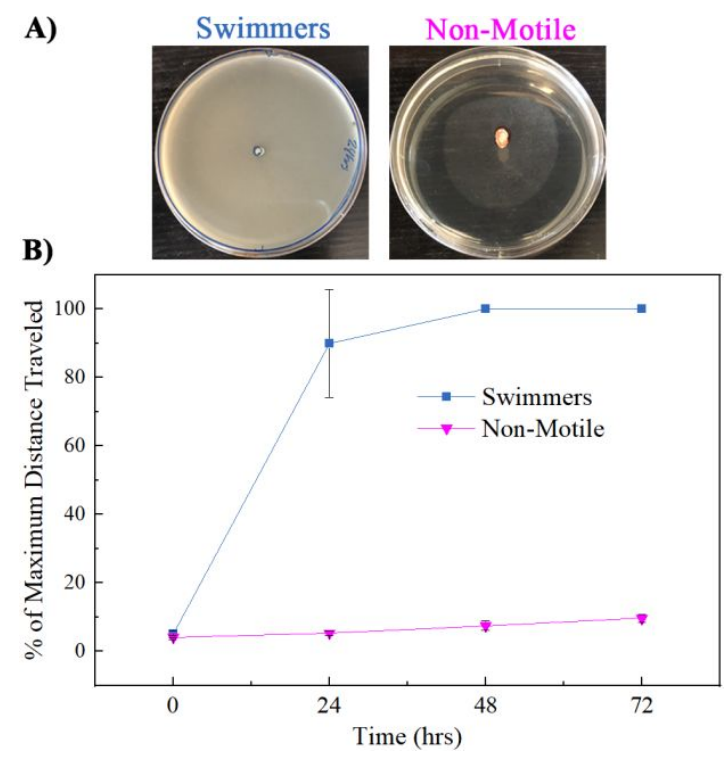

Figure SI-1. Motility assay for swimming and non-motile strains.

A) Images of plates after 72 hours of incubation. B) Time dependence of colony expansion. 


\section{Calculations for Velocity of a Neutrally-Buoyant Sphere near a Wall in Shear Flow}

The analytical model of Goldman, Cox, and Brenner, "GCB," was employed to calculate the velocity of a neutrally-buoyant sphere near a wall in shear flow. The model includes both near and far-field solutions of the equations of motion, and employs $a$ for particle radius, $h$ for the separation of the particle's center from the surface and "gap" for the closest distance of the particle surface to the wall, as illustrated. In the current work, when discussing particle sizes, nominal length scales such as " 2 micron particles" refer to particles of a 2 micron diameters so, for example, $a=1 \mu \mathrm{m}$.

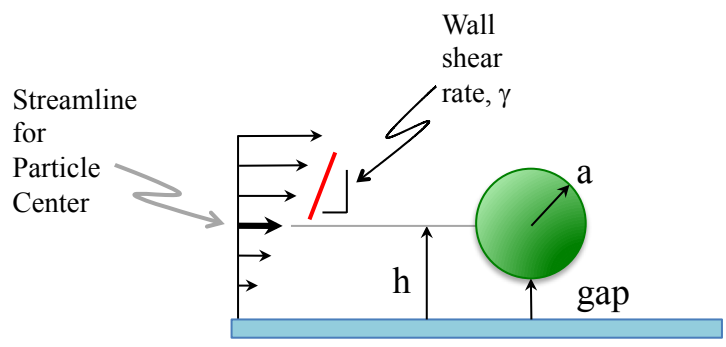

Figure SI 2. Schematic of geometry for a sphere of radius $a$, flowing near a wall. The streamline corresponding to the particle's center is highlighted. The wall shear rate, $\gamma$, is the gradient of the velocity near the wall, as shown.

Far field hydrodynamics. For particles far from a wall, GCB Equation 4.8a was employed to calculate the particle velocity, normalized by the streamline velocity corresponding to the particle's center, for different particle heights from the wall, summarized in Figure SI-3. Figure SI-3A shows the normalized particle velocity in terms of the distance between a particle's center and the wall, while Figure SI-3B shows the same calculation but in terms of the gap between the particle's surface and the wall.

A)

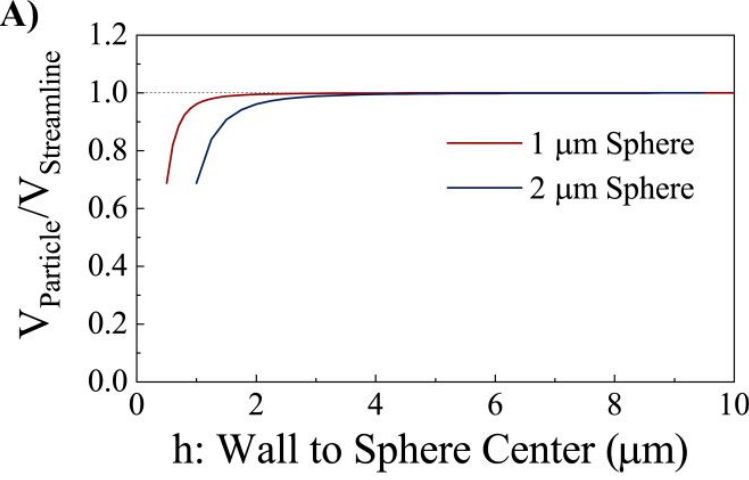

B)

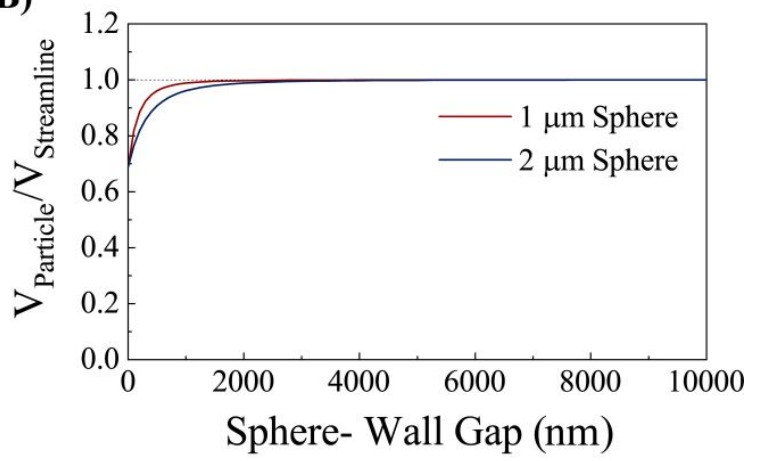

Figure SI 3. Calculations from GCB equation 4.8 showing particle velocity normalized by the streamline velocity corresponding to the particle's center, for 1 and $2 \mu \mathrm{m}$ diameter particles. (A) as a function of particle height from surface (B) as a function of the gap between the particle's surface and the wall for the same of particle heights. 
Figure SI-3 shows that particles travel at the velocity equal to the unperturbed streamline velocity corresponding to each particle's center as long as the particle is more than a diameter from the wall. Even when particles approach more closely, the unperturbed streamline velocity matches the particle velocity, within no more than $10 \%$ error for particle-wall gaps greater than $20 \%$ of the particle's diameter. Thus, a $2-\mu \mathrm{m}$ sphere deviates from its free stream velocity only when it approaches within $400 \mathrm{~nm}$ of a wall, and a $1-\mu \mathrm{m}$ sphere deviates from its free stream velocity only when it approaches within $200 \mathrm{~nm}$ of a wall.

Near field hydrodynamics. Figure SI-4 further considers the velocities of spheres close to the wall, plotting both the near field (GCB 4.11) and far field (GCB4.8a) solutions together.

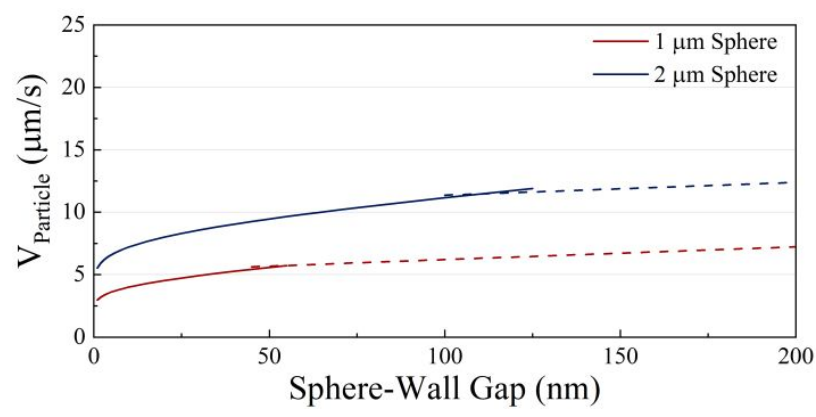

Figure SI 4. Calculations from GCB showing particle velocity as a function the gap between the particle surface and the wall, for 1 and $2 \mu \mathrm{m}$ diameter particles. The wall shear rate is $15 \mathrm{~s}^{-1}$. The solid lines are the nearfield solutions, GCB equation 4.11 while the dashed lines are the far field solutions, GCB 4.8a. 


\section{Motivation for choice to study cells less moving more slowly than $9.3 \mu \mathrm{m} / \mathrm{s}$}

Figure SI-4 shows that for neutrally buoyant spheres near a rigid wall, measurements of velocity provide precise information about the distance of the particle from the wall. Relevant to our work, with a sphere providing a crude approximation of a capsular cell body, particle motion less than $15 \mu \mathrm{m} / \mathrm{s}$ corresponds to particle-wall gaps of $200 \mathrm{~nm}$ or less, depending on particle size in the 1-2 $\mu \mathrm{m}$ size range.

To the extent that, during near-surface travel in shear, long range hydrodynamics orient capsules and suppresses tumbling, ${ }^{4}$ the smaller $1 \mu \mathrm{m}$ diameter spheres are included in calculations. The results motivate the choice of cells traveling more slowly than $10 \mu \mathrm{m} / \mathrm{s}$ in studies of surface-influenced travel. Notably, for $1 \mu \mathrm{m}$ diameter spheres traveling at 4,6 , and $8 \mu \mathrm{m} / \mathrm{s}$, the gaps between the particle and the surface are 6,40 and $93 \mathrm{~nm}$, respectively. Two micron spheres traveling at the same velocities have much smaller gaps with the wall, down to a single nanometer. For instance, the sphere-wall gap for a 2 $\mu \mathrm{m}$ sphere traveling at $9.3 \mu \mathrm{m} / \mathrm{s}$ is $25 \mathrm{~nm}$. The use of a sphere to approximate a capsular cell body is employed here only as means to estimate separation between the cell and the chamber wall, and has been employed in prominent treatments of swimming E. coli. ${ }^{5}$ Any error in the cell-wall separation in these calculations does not propagate into other parts of the work.

\section{Impact of choice of velocity threshold on results}

In the main paper, a velocity threshold of $9.3 \mu \mathrm{m} / \mathrm{s}$ for a distance of 5 microns was chosen as a criteria for surface associated travel. This corresponded to a duration of surface associated travel of at least $0.6 \mathrm{~s}$. Cells meeting this criterion at some point within the observation window were further analyzed and included in the various distributions of Figures 4-6 in the main paper.

This section of the Supporting Information examines the consequences of choosing a different threshold. We show that while some of the distributions look slightly different for different thresholds, as they must, the overall shapes of the distributions, their peaks, and the impact of the cell-coating combinations are not affected.

The threshold of $11.3 \mu \mathrm{m} / \mathrm{s}$ was chosen, here as an exercise, for cells traveling a distance of $4.5 \mu \mathrm{m}$, now including data with a lower minimum engagement time of $0.6 \mathrm{~s}$. The velocity of $11.3 \mathrm{um} / \mathrm{s}$ corresponds to cell-wall gap of $62 \mathrm{~nm}$, with the cell modeled as a $2 \mu \mathrm{m}$ sphere. Data groups, which now include greater numbers of engagements due to the broader definition of engagement were reanalyzed. A comparison results based on a criteria of 9.3 or $11.3 \mu \mathrm{m} / \mathrm{s}$ and a comparison is presented here.

Figure SI-5 illustrates the ultimate negligible impact of the choice of velocity cut off, 9.3 or $11.3 \mu \mathrm{m} / \mathrm{s}$, on the distribution of cells returning to the end-tethered PEG coating for repeat engagements, the velocity distribution, and the overall residence time. Here swimmers and control non-motile cells are compared. Of note there must be some impact of the threshold, for instance introducing additional cells into the distribution with when a broader criterion $(11.3 \mathrm{um} / \mathrm{s})$ is applied, and increasing the range of distribution of surface residence times. Important features such as the shapes of the distribution and the difference between swimmers and control non-motile cells are preserved. 

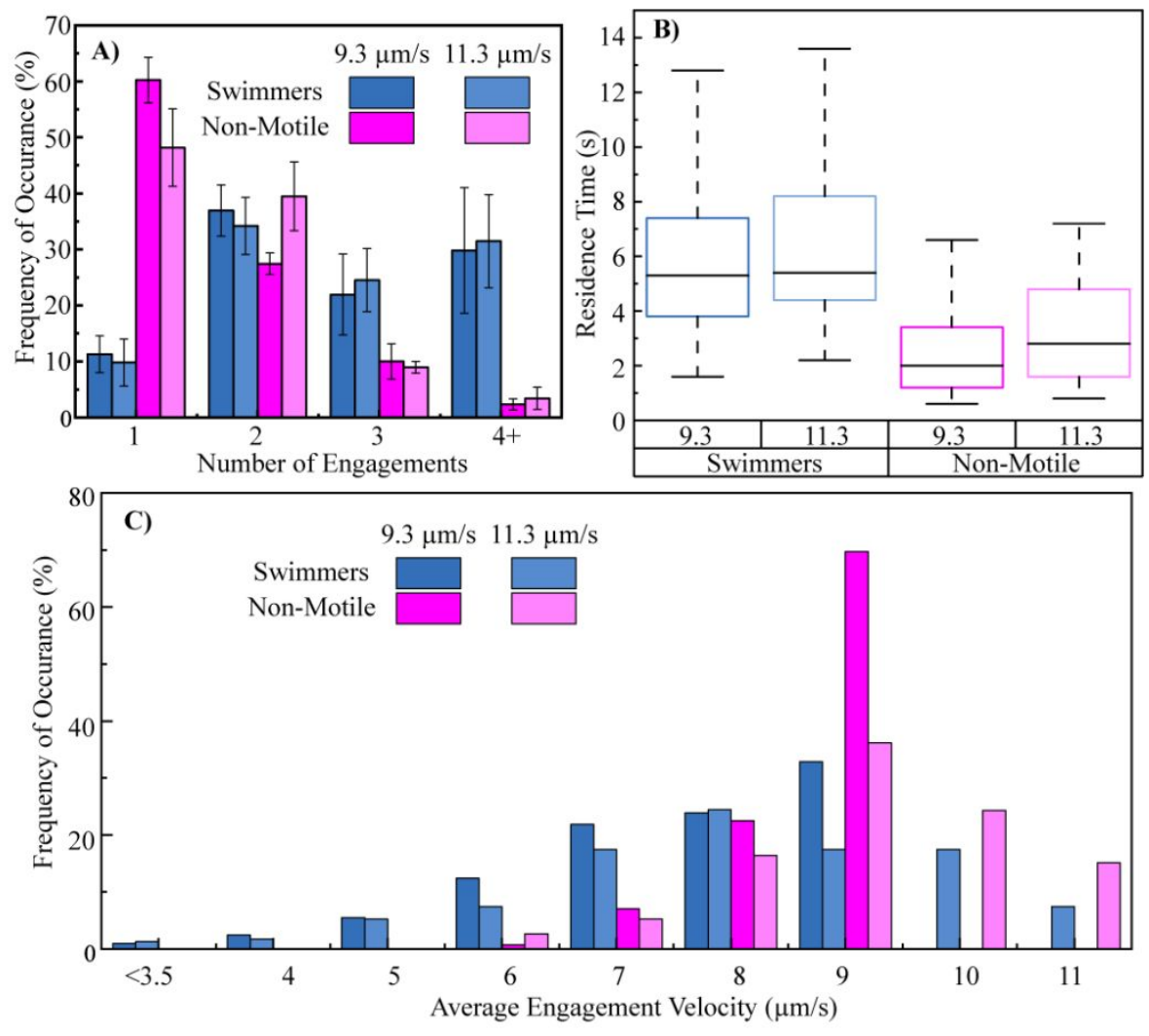

Figure SI-5. Comparison of the quantities measured with velocity cut offs of 9.3 versus $11.3 \mu \mathrm{m} / \mathrm{s}$ for swimmers and non-motile cells on an end-tethered PEG layer. (A) number of engagements per cell for cells having at least one engagement, (B) overall cell residence time in the field of view, and (C) average engagement velocities. Swimmers are in blue and non-motile controls are in pink. 


\section{Estimated Force of a Swimming E. Coli Cell}

Swimming into a surface. Chattopadhyay et al. ${ }^{6}$ employed a laser trap to measure the force of swimming E. coli HCB30 cells. They report a forward thrust force of $0.57 \mathrm{pN}$, averaged from measurements on 200 cells. With an approximate cross sectional length of $0.75 \mu \mathrm{m}$, a bacterium swimming into a surface would exert a local normal stress, $\sigma=$ thrust $/(\text { cross sectional length })^{2}$ of $1 \mathrm{~Pa}$ against a surface. We employ this value as an order of magnitude estimate.

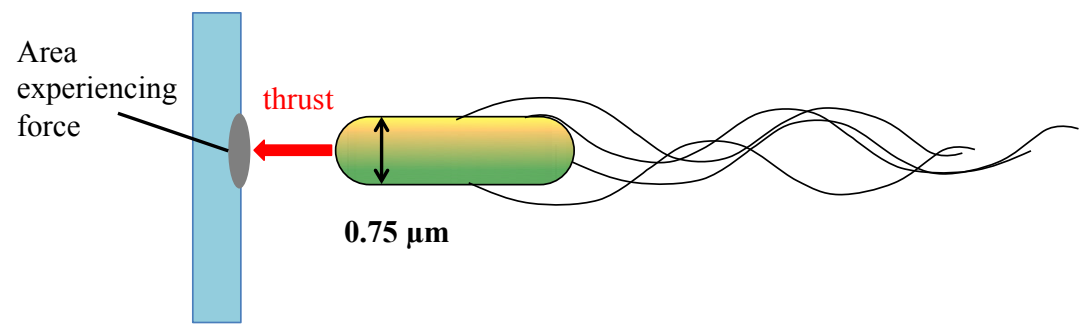

Figure SI 6. Geometry for a cell swimming into the surface.

Swimming along a surface. Chattopadhyay et al. ${ }^{6}$ also report a flagellar torque of $5 \times 10^{-19} \mathrm{Nm}$. With a swimming stroke radius on the order of $0.5 \mu \mathrm{m}$, a single flagella impinging on a surface as the cell swims along might impose a local normal stress, $\sigma=$ (torque/ radius) $/$ contact area $=1 \mathrm{pN} /\left(0.02 \mu \mathrm{m}^{2}\right)=50 \mathrm{~Pa}$, where there is a very small contact area of $20 \mathrm{~nm}$ (the flagellar diameter) by $1 \mu \mathrm{m}$ (a contact length) for a segment of one flagella.
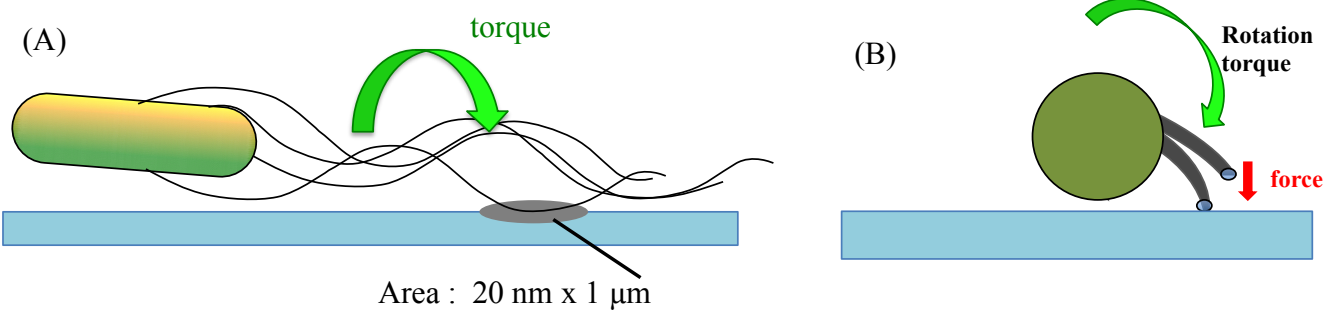

Figure SI 7. Side of rotating flagella impinges on surface behind a swimming cell, but approximately normal to the surface. Right: Cutaway view behind swimming cell, showing two of the flagella from the bundle, as they impinge on the surface, with an initial contact normal to the surface.

\section{Possible Coating Deformation or Penetration}

The collision stresses of the bacterial head and single flagella represent maximum values and are small compared with the reported stiffnesses of the PEG coatings in Table I. Also a point of comparison, the wall stress for the flow of water at a wall shear rate of $15 \mathrm{~s}^{-1}$ is $0.0135 \mathrm{~Pa}$, two orders of magnitude smaller than the local stress from bacterial collisions. 
The strain, $\varepsilon$, is a measure of deformation $\Delta \mathrm{L}$ resulting from applied stress, normalized by the length in unstressed state, $\mathrm{L}_{\mathrm{o}}: \varepsilon=\Delta \mathrm{L} / \mathrm{L}_{\mathrm{o}}$. An estimate of the local coating strain, $\varepsilon$, produced by a bacterial collision follows as $\varepsilon=\sigma$ /modulus. $\sigma=1 \mathrm{~Pa}$ for a head on collision yields $\varepsilon=2 \times 10^{-6}$ and $\varepsilon=8 \times 10^{-7}$ for the tethered PEG layer and stiff hydrogel respectively. The expected local strain on the soft hydrogel by contrast is $\varepsilon=1 \times 10^{-4}$. Only for the soft hydrogel, many microns thick, might a nanometric deformation result from a head-on bacterial collision. To the extent that a flagellar collision occurs over a smaller contact area to produce a local stress 1-2 orders of magnitude greater than the "head-on" collision, it is certainly reasonable that the soft coating would experience strains of $\varepsilon=5 \times 10^{-3}$. For coating thicknesses of hundreds of microns, the deformation could locally be on the order of the thickness of a single flagellum, $20 \mathrm{~nm}$.

\section{Statistical Analysis}

Tables SI-1 through SI-3 summarize t-tests run on data that was plotted in Figures 4 and 5 of the main paper.

Table SI-1 Average Engagement Velocity -Figure 4A,B

\begin{tabular}{|c|c|c|c|c|c|c|c|}
\hline \multirow{2}{*}{\multicolumn{2}{|c|}{$p=$}} & \multicolumn{3}{|c|}{ Swimmers } & \multicolumn{3}{|c|}{ Non-Motile } \\
\hline & & \multirow[t]{2}{*}{ Tethered } & \multirow{2}{*}{$\frac{\text { Stiff Gel }}{7.02 \mathrm{E}-11}$} & \multirow{2}{*}{$\frac{\text { Soft Gel }}{6.89 \mathrm{E}-06}$} & \multirow{2}{*}{$\begin{array}{l}\text { Tethered } \\
1.12 \mathrm{E}-16\end{array}$} & \multirow{2}{*}{$\frac{\text { Stiff Gel }}{1.22 \mathrm{E}-14}$} & \multirow{2}{*}{$\frac{\text { Soft Gel }}{1.84 \mathrm{E}-16}$} \\
\hline$\infty$ & Tethered & & & & & & \\
\hline \multirow{2}{*}{ 范 } & Stiff Gel & 7.018E-11 & & 0.165773 & $5.24 \mathrm{E}-05$ & 0.005915 & 0.000144 \\
\hline & Soft Gel & 6.887E-06 & 0.165773 & & $3.63 \mathrm{E}-05$ & 0.001683 & $7.75 \mathrm{E}-05$ \\
\hline \multirow{3}{*}{$\begin{array}{l}\stackrel{0}{E} \\
\sum_{0}^{0} \\
\vdots \\
0 \\
z\end{array}$} & Tethered & $1.125 \mathrm{E}-16$ & $5.24 \mathrm{E}-05$ & $3.63 \mathrm{E}-05$ & & 0.048997 & 0.357381 \\
\hline & Stiff Gel & $1.218 \mathrm{E}-14$ & 0.005915 & 0.001683 & 0.048997 & & 0.095016 \\
\hline & Soft Gel & $1.842 \mathrm{E}-16$ & 0.000144 & $7.75 \mathrm{E}-05$ & 0.357381 & 0.095016 & \\
\hline
\end{tabular}

$\mathrm{p}>0.1=$ n.s., $\mathrm{p}<0.1=*, \mathrm{p}<0.05=* *, \mathrm{p}<0.01=* * *$ 
Table SI-2 Residence Time Per Engagement -Figure 4 C,D

\begin{tabular}{|c|c|c|c|c|c|c|c|}
\hline \multirow{2}{*}{\multicolumn{2}{|c|}{$\mathrm{p}=$}} & \multicolumn{3}{|c|}{ Swimmers } & \multicolumn{3}{|c|}{ Non-Motile } \\
\hline & & Tethered & Stiff Gel & Soft Gel & Tethered & Stiff Gel & Soft Gel \\
\hline \multirow{3}{*}{ 离 } & Tethered & & 0.028397 & 0.001109 & $2.28 \mathrm{E}-05$ & $5.18 \mathrm{E}-09$ & $3.21 \mathrm{E}-15$ \\
\hline & Stiff Gel & 0.0283971 & & $4.16 \mathrm{E}-06$ & 7.22E-09 & $2.64 \mathrm{E}-12$ & $6.34 \mathrm{E}-18$ \\
\hline & Soft Gel & 0.0011089 & 4.16E-06 & & 0.374266 & 0.025242 & $2.48 \mathrm{E}-05$ \\
\hline \multirow{3}{*}{ 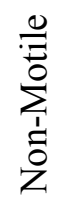 } & Tethered & $2.285 \mathrm{E}-05$ & 7.22E-09 & 0.374266 & & 0.019603 & $9.4 \mathrm{E}-07$ \\
\hline & Stiff Gel & $5.18 \mathrm{E}-09$ & $2.64 \mathrm{E}-12$ & 0.025242 & 0.019603 & & 0.000937 \\
\hline & Soft Gel & $3.21 \mathrm{E}-15$ & $6.34 \mathrm{E}-18$ & $2.48 \mathrm{E}-05$ & $9.4 \mathrm{E}-07$ & 0.000937 & \\
\hline
\end{tabular}

Table SI-3 Maximum Separation Velocity -Figure 5C,D

\begin{tabular}{|c|c|c|c|c|c|c|c|}
\hline \multirow{2}{*}{\multicolumn{2}{|c|}{$\mathrm{p}=$}} & \multicolumn{3}{|c|}{ Swimmers } & \multicolumn{3}{|c|}{ Non-Motile } \\
\hline & & Tethered & Stiff Gel & Soft Gel & Tethered & Stiff Gel & Soft Gel \\
\hline \multirow{3}{*}{ 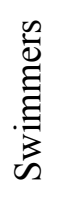 } & Tethered & & 0.33075 & 0.0798 & $2.37 \mathrm{E}-05$ & 0.000346 & $5.22 \mathrm{E}-06$ \\
\hline & Stiff Gel & 0.33075 & & 0.353409 & 0.054616 & 0.100416 & 0.039146 \\
\hline & Soft Gel & 0.0798 & 0.353409 & & 0.001457 & 0.02518 & 0.000371 \\
\hline \multirow{3}{*}{$\begin{array}{l}\stackrel{0}{\Xi} \\
\sum_{0}^{0} \\
\dot{0} \\
z\end{array}$} & Tethered & $2.372 \mathrm{E}-05$ & 0.054616 & 0.001457 & & 0.077761 & 0.309516 \\
\hline & Stiff Gel & 0.0003456 & 0.100416 & 0.02518 & 0.077761 & & 0.027366 \\
\hline & Soft Gel & $5.221 \mathrm{E}-06$ & 0.039146 & 0.000371 & 0.309516 & 0.027366 & \\
\hline
\end{tabular}

$$
\mathrm{p}>0.1=\text { n.s., } \mathrm{p}<0.1=*, \mathrm{p}<0.05=* *, \mathrm{p}<0.01=* * *
$$




\section{References}

1. Kearns, D. B. A field guide to bacterial swarming motility. Nat. Rev. Microbiol. 2010, 8, 634-644.

2. Swiecicki, J. M.; Sliusarenko, O.; Weibel, D. B. From swimming to swarming: Escherichia coli cell motility in two-dimensions. Integr. Biol. 2013, 5, 1490-1494.

3. Goldman, A. J.; Cox, R. G.; Brenner, H. Slow Viscous Motion of a Sphere Parallel to a Plane Wall. 2. Couette Flow. Chem. Eng. Sci. 1967, 22, 653-660.

4. Kaya, T.; Koser, H. Characterization of Hydrodynamic Surface Interactions of Escherichia coli Cell Bodies in Shear Flow. Phys. Rev. Lett. 2009, 103.

5. Lauga, E.; DiLuzio, W. R.; Whitesides, G. M.; Stone, H. A. Swimming in circles: Motion of bacteria near solid boundaries. Biophys. J. 2006, 90, 400-412.

6. Chattopadhyay, S.; Moldovan, R.; Yeung, C.; Wu, X. L. Swimming efficiency of bacterium Escherichia coli. Proc. Natl. Acad. Sci. U. S. A. 2006, 103, 13712-13717. 